\title{
Growth inhibition of estrogen receptor positive human breast cancer cells by Taheebo from the inner bark of Tabebuia avellandae tree
}

\author{
B. MUKHERJEE ${ }^{1}$, N. TELANG ${ }^{2}$ and G.Y.C. WONG ${ }^{1}$ \\ ${ }^{1}$ Strang Cancer Prevention Center, New York, NY; ${ }^{2}$ Palindrome Liaisons, Montvale, NJ, USA
}

Received February 26, 2009; Accepted May 8, 2009

DOI: 10.3892/ijmm_00000228

\begin{abstract}
Selective estrogen receptor (ER) modulators are used as a therapy for $\mathrm{ER}^{+}$clinical breast cancer, but they exhibit adverse effects. Herbal medicines may provide an alternative or complementary approach. Taheebo, extracted from the inner bark of the Tabebuia avellandae tree found in the Brazilian Amazon, exhibits selective anti-proliferative effects in carcinoma cell lines. The present study identifies the mechanistic leads for the inhibitory effects of Taheebo. Human breast carcinoma derived $\mathrm{ER}^{+} \mathrm{MCF}-7$ cells were used as the model. Aqueous extract of Taheebo was the test compound. Cell cycle analysis, clonogenic assay, and global gene expression profiles were the quantitative parameters. Taheebo treatment resulted in a dose/time-dependent growth inhibition ( $\mathrm{S}$ phase arrest, reduced clonogenecity) and initiation of apoptosis (chromatin condensation). A 6-h treatment with $1.5 \mathrm{mg} / \mathrm{ml}$ Taheebo modulated the gene expression of G2 specific cyclin B1 (-2.0-fold); S phase specific PCNA (-2.0-fold) and OKL38 (+11.0-fold); apoptosis specific GADD-45 family $(+1.9-5.4$-fold $)$, Caspases (+1.6-1.7-fold), BCL-2 family (-1.5-2.5-fold), estrogen responsive ESR1 (-1.5-fold), and xeno-biotic metabolism specific CYP 1A1 (+19.8 fold) and CYP 1B1 (+7.9fold). The anti-proliferative effects of Taheebo correlate with down-regulated cell cycle regulatory and estrogen responsive genes, and up-regulated apoptosis specific and xeno-biotic metabolism specific genes. These data validate a rapid mechanistic approach to prioritize efficacious herbal medicines, thereby complementing the existing endocrine therapy for breast cancer.
\end{abstract}

\section{Introduction}

It is well recognized that $\sim 80 \%$ of sporadic clinical breast cancers express estrogen receptor (ER) and that endocrine therapy with or without chemotherapy is a common treatment

Correspondence to: Dr Nitin Telang, Cancer Prevention Research, Palindrome Liaisons, 10 Rolling Ridge Road, Suite B, Montvale, NJ 07645-1559, USA

E-mail: entitytoo@cs.com

Key words: human breast carcinoma cells, growth inhibition, global gene expression profile option $(1,2)$. However, long-term treatment with chemoendocrine therapy is frequently associated with adverse toxicity that compromises patient compliance $(3,4)$.

In recent years complementary and alternative approaches utilizing herbal medicines are being extensively used independently, or as adjuvants to chemo-endocrine therapy in an effort to reduce therapy related toxicity and enhance efficacy (3,5-8). However, long-term safety, efficacy and toxicity of herbal medicines and their interactions with conventional therapeutic regimen remain to be established.

Taheebo, a naturally occurring constituent of the inner bark of the Tabebuia avellandae tree found in the Brazilian Amazon, has been used for over 1,500 years in South America to treat a variety of health conditions (9-11). In the USA, Taheebo is available as a nutritional supplement under the name Pau d'Arco tea. However, the mechanism(s) of action, molecular targets of efficacy and long-term toxicity profile of Taheebo remain to be identified.

The human breast carcinoma derived MCF-7 cell line represents a widely used preclinical cell culture model for hormone responsive $\mathrm{ER}^{+}$clinical breast cancer. This model has been extensively utilized in in vitro (cell culture) as well as in vivo (xeno-transplant) approaches to evaluate the efficacy of selective estrogen receptor modulators and aromatase inhibitors for preventive/therapeutic intervention of hormone responsive $\mathrm{ER}^{+}$clinical breast cancer $(12,13)$.

The experiments in the present study utilized the MCF-7 cell culture model to examine the growth inhibitory effects of Taheebo and identify possible mechanistic leads for its efficacy. The phenomenological and mechanistic data generated from this study provide evidence that the growth inhibitory effects of Taheebo are associated with down-regulated expression of several cell cycle regulatory and estrogen responsive genes, and with up-regulated expression of apoptosis specific genes and of xeno-biotic metabolism specific genes. Taken together, the outcome of this study validates the present experimental approach for a mechanism based evaluation of the efficacy of plant derived medicinal products for therapy of hormone responsive $\mathrm{ER}^{+}$breast cancer.

\section{Material and methods}

$E R^{+}$human mammary carcinoma MCF-7 cell line. The $\mathrm{ER}^{+}$ human mammary carcinoma derived MCF-7 cell line was 
obtained from ATCC, Manassas, VA. The cell line was maintained and propagated in MEM supplemented with $10 \%$ fetal bovine serum, $2 \mathrm{mM}$ l-glutamine, $10 \mu \mathrm{g} / \mathrm{ml}$ bovine insulin and $1 \%$ penicillin-streptomycin mixture as recommended by the supplier. Routinely, cells were maintained at $37^{\circ} \mathrm{C}$ in a humidified atmosphere of $5 \% \mathrm{CO}_{2}$ and $95 \%$ air, and were subcultured at about $70 \%$ confluency.

Dose response of Taheebo. Lyophilized powder of nonfractionated Taheebo was provided by Taheebo Japan Co. Ltd. (Osaka, Japan). This powder was dissolved in double distilled water at a concentration of $500 \mathrm{mg} / \mathrm{ml}$ to make the stock solution. This stock solution was serially diluted in the MEM culture medium to obtain various concentrations of Taheebo ranging from $1.5,0.75,0.5,0.25,0.125$ and $0.05 \mathrm{mg} / \mathrm{ml}$ for dose response experiments. The initial dose response of a 6day duration was conducted at the concentration range of $0.05-0.5 \mathrm{mg} / \mathrm{ml}$. The viable cell counts were obtained using a hemocytometer after trypan blue staining and were confirmed using a coulter counter. For the experiments on cell cycle analysis, induction of apoptosis/necrosis and clonogenecity Taheebo was evaluated at the concentration of $0.75 \mathrm{mg} / \mathrm{ml}$. For micro-array based gene expression profiles and electron microscopy Taheebo was evaluated at a concentration of $1.5 \mathrm{mg} / \mathrm{ml}$ for durations of 6 and $24 \mathrm{~h}$, respectively.

Cell cycle progression. For cell cycle analysis, controls and Taheebo treated cultures were trypsinized and washed with PBS. The cell suspensions were fixed in $75 \%$ ethanol and stained with propidium iodide (PI). The fluorescence assisted cell sorting was performed using a flow cytometer (BD FACS Canto, Becton Dickenson), and percent distribution of cell population in $\mathrm{G} 0 / \mathrm{G} 1, \mathrm{~S}$, and $\mathrm{G} 2 / \mathrm{M}$ phases of cell cycle were calculated using the multicycle software. The data were expressed as $\mathrm{G} 1: \mathrm{S}+\mathrm{G} 2 / \mathrm{M}$ ratio.

Electron microscopy for apoptosis and necrosis. MCF-7 cells treated with $1.5 \mathrm{mg} / \mathrm{ml}$ of Taheebo for $24 \mathrm{~h}$ and parallel nontreated controls were fixed with $2.5 \%$ Glutaraldehyde in $0.1 \mathrm{M}$ sodium cacodylate buffer $(\mathrm{pH} 7.4)$ for $1 \mathrm{~h}$ at room temperature, rinsed in the same buffer several times, post-fixed in $1 \%$ osmium tetroxide solution, dehydrated in serial ethanol dilutions and embedded in Poly-Bed araldite media. Ultrathin sections $(65-70 \mathrm{~nm})$ were then counterstained with uranyl acetate and lead citrate and viewed under a Philips-410 transmission electron microscope (Phillips, Eindoven, Holland).

Clonogenic assay. This assay was performed using a protocol similar to the one previously published (14). Briefly, MCF-7 cells were pre-treated with Taheebo at a concentration of 0.75 $\mathrm{mg} / \mathrm{ml}$ for $72 \mathrm{~h}$. The treated cells were trypsinized, washed and re-plated at a seeding density of 1,000 cells per flask. The untreated control cells were also re-plated at an identical seeding density. Both sets of cultures were maintained without any further treatment for 8 days. Percentage of survival was calculated as (colony number in treated group/ colony number in control group) x 100 .

Micro-array analysis for global gene expression profile. Cells (1.0x10 cells per flask) were plated in T25 flasks $24 \mathrm{~h}$ prior to treatment. Treatment with Taheebo was performed for $6 \mathrm{~h}$ at a dose of $1.5 \mathrm{mg} / \mathrm{ml}$. RNA was isolated following the protocol for RNeasy kit (Qiagen Inc., Chatsworth, CA). The RNA was quantified using a spectrophotometric assay by absorbance at $260 \mathrm{nM}$. The integrity of RNA was evaluated by, i) $260: 280$ absorbance ratio, ii) $28 \mathrm{~S}: 18 \mathrm{~S}$ intensity ratio, and iii) RNA integration number (RIN) using the Bioanalyzer (Agilent Technologies). RNA was amplified using a low input linear amplification kit according to the process outlined by the manufacturer (Agilent Technologies). Amplified target cRNA (1-5 $\mu \mathrm{g}$ ) was labeled with either cyanine-5 or cyanine-3 using ULS RNA Fluorescent Labeling Kit (Kreatech Biotechnology, Amsterdam, The Netherlands), according to the manufacturer's protocol. Concentration of labeled cRNA and the label incorporation was determined with a Nanodrop-1000 spectrophotometer. All labeling and post-labeling procedures were conducted in an ozone-free enclosure to ensure the integrity of the label. Labeled materials were setup for fragmentation reaction as described by the Agilent Technology Protocol processing manual and hybridized overnight in a rotating oven at $65^{\circ} \mathrm{C}$ in an ozonefree room. Whole Human Genome slide $4 \mathrm{x} 44 \mathrm{~K}$ format (Agilent Technologies) was used. From each labeled sample, $825 \mathrm{ng}$ were co-hybridized using Sure-Hyb chambers (Agilent Technologies). Wash conditions used were as outlined in the Agilent Technologies processing manual, and the arrays were scanned using Agilent scanner 2505B. Feature Extraction from Agilent Technologies was used to extract the microarray data. Further analysis was performed using Rosetta luminator software by MOgene, LC, St. Louis, MO.

Real-time PCR for mico-array data validation. The RNA was used to synthesize complementary DNA using TaqMan one-step RT-PCR master Mix Reagents (Applied Biosystems, Foster City, CA) with Taq Man probe-primers set (Applied Biosystems, Foster City, CA).

Real-time PCR was used to verify the micro-array data for six modulated genes using the following Taq Man probes and primers for individual genes (Applied Biosystems, Foster City, CA). DUSP 4 (NM_001394, Assay ID Hs 00154826_m1), DUSP 10 (NM_007207, Assay ID Hs 00200527_m1), GTSE 1 (NM_016426_m1, Assay ID Hs 00212681_m1), Cyclin B1 (NM_031966, Assay ID Hs 00259126_m1), CYP 1A1 (NM_000499, Assay ID Hs 00153120_m1) and OKL 38 (NM_013370, Assay ID Hs 00203539_m1). Briefly, the reverse transcription protocol used $1 \mu \mathrm{g}$ of total RNA and random hexamer method. The fold change in expression of each gene was calculated using the $\Delta \Delta C \mathrm{C}$ method with GAPDH mRNA as the internal control (15).

Statistical analysis. Mean data with their deviations $( \pm \mathrm{SEM})$ are presented from independent triplicate sets of experiments. $\mathrm{P}$-values were calculated using two sample t-test and $\mathrm{p}<0.05$ was considered significant.

The statistical analysis of the data from micro-array was performed on three independent sets of experiments using Agilent and Rosetta software (MOgene, LC). Differentially expressed genes were considered as average of three sets of experiments. Modulations of $\geq 1.5$-fold change (log ratio $\geq+0.176$ or $\leq-0.176$ ) in the Taheebo treated group compared 
A
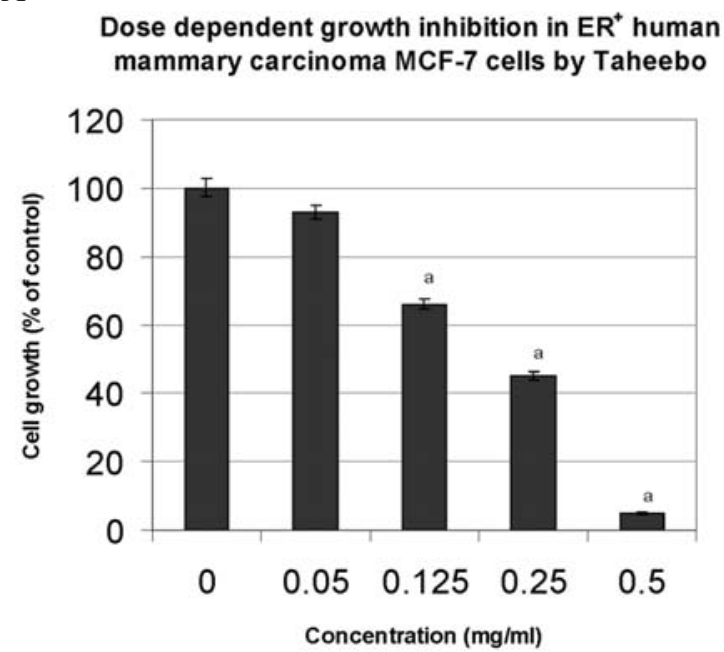

B

Time dependent growth inhibition in $\mathrm{ER}^{+}$human mammary carcinoma MCF-7 cells by Taheebo

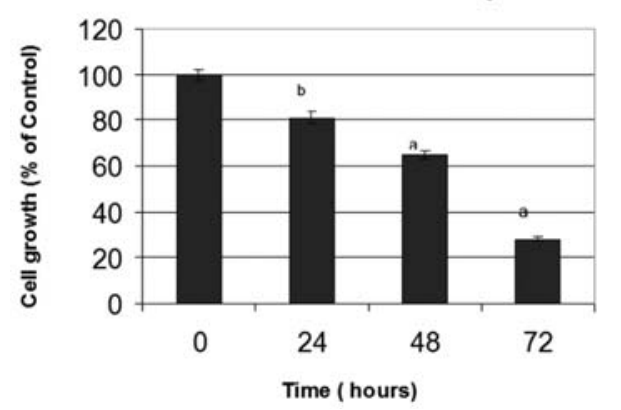

Figure 1. Dose-dependent growth inhibition of $\mathrm{ER}^{+} \mathrm{MCF}-7$ cells by Taheebo (A) Inhibition of growth in response to treatment with $0.05-0.5 \mathrm{mg} / \mathrm{ml} \mathrm{of}$ Taheebo. Data expressed as inhibition of cell growth (\% control). Mean \pm SEM, $\mathrm{n}=3$ per treatment group. $\mathrm{P}<0.001$ compared to control. (B) Short term dose response to growth inhibition by $0.75 \mathrm{mg} / \mathrm{ml}$ Taheebo at 24,48 , and $72 \mathrm{~h}$ post-seeding. Data expressed as inhibition of cell growth (\% control). Mean \pm SEM, $\mathrm{n}=3$ per treatment group. $\mathrm{P}<0.01$ compared to control.

to the untreated control group were considered statistically significant $(\mathrm{p}<0.01)$.

\section{Results}

Dose response of Taheebo on $E R^{+} M C F-7$ cells. The data presented in Fig. 1A exhibits dose-dependent growth inhibition of MCF-7 cells in response to treatment with Taheebo. The $\mathrm{IC}_{50}$ concentration extrapolated from growth curves after a 6-day treatment schedule was $\sim 0.25 \mathrm{mg} / \mathrm{ml}$.

The short-term dose response of high dose $(0.75 \mathrm{mg} / \mathrm{ml})$ Taheebo at 24, 48 and $72 \mathrm{~h}$ post-seeding demonstrated 20, 35 and $72 \%$ growth inhibition relative to that of controls (Fig. 1B). These data identified the optimal concentration of Taheebo for subsequent experiments.

To identify minimum effective, maximum cytostatic and toxic concentrations of Taheebo, a time-dependent kinetics of viable cell number was preformed. This experiment revealed that a $24-\mathrm{h}$ treatment with $1.5 \mathrm{mg} / \mathrm{ml}$ of Taheebo resulted in a statistically non-significant $15 \%$ increase in the viable cell number relative to the initial seeding density of $1.0 \times 10^{5}$ cells.

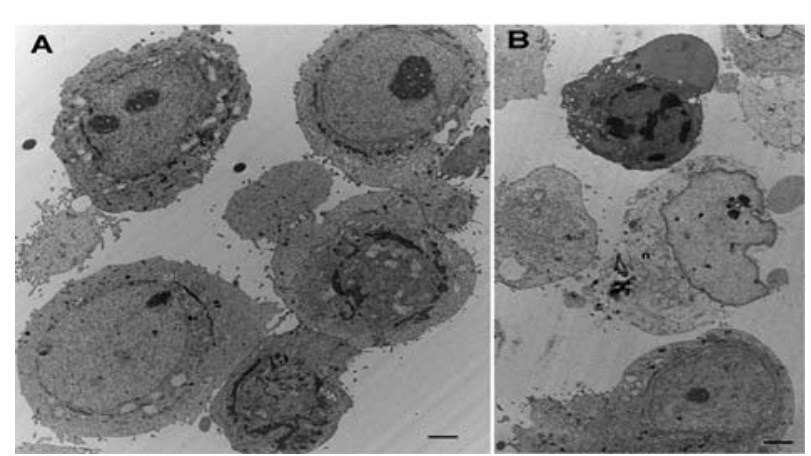

Figure 2. Transmission electron microscopy based cellular morphology of apoptotic and necrotic cells. (A) Untreated control, (B) treated with $1.5 \mathrm{mg} / \mathrm{ml}$ of Taheebo for $24 \mathrm{~h}$. Note the presence of peripheral condensation and central fragmentation of nuclear chromatin in the apoptotic cell, and the presence of membrane blebbing and cytoplamic lysosomes in the necrotic cells.

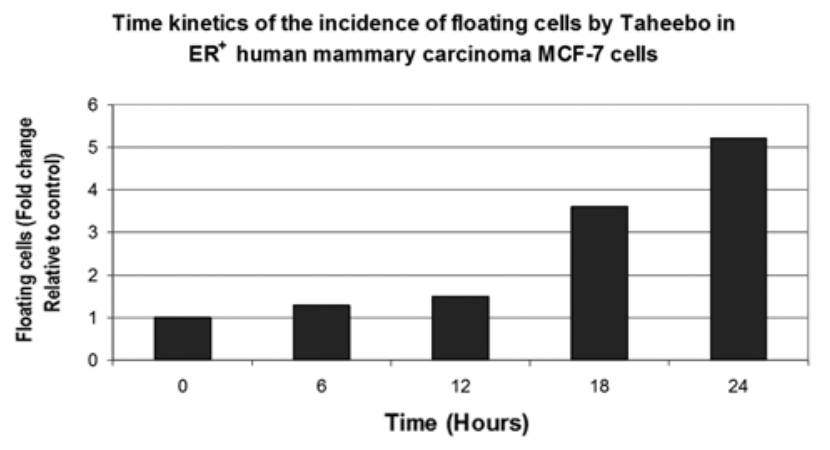

Figure 3. Time-dependent increase in non-adherent (floating) cells in response with the treatment of $1.5 \mathrm{mg} / \mathrm{ml}$ of Taheebo.

In contrast, a 48 -h treatment with $1.5 \mathrm{mg} / \mathrm{ml}$ Taheebo resulted in a $94 \%$ decrease in the viable cell number relative to the initial seeding density. Thus, the 24-h treatment was defined as non-effective, while the 48 -h treatment was defined as toxic.

Cell cycle regulation of $E R^{+} M C F-7$ cells by Taheebo. The time-dependent alteration in cell cycle regulation of MCF-7 cells in response to treatment with $0.75 \mathrm{mg} / \mathrm{ml}$ Taheebo is presented in Table I. The data expressed as the G1:S+G2/M ratio demonstrate a time-dependent progressive decrease associated with the $\mathrm{S}$ phase arrest of cells.

Induction of apoptosis/necrosis in $\mathrm{ER}^{+} \mathrm{MCF}-7$ cells by Taheebo. The effect of Taheebo on cell viability was also evaluated by transmission electron microscopy based cellular morphology (Fig. 2A and B), and by the incidence of nonviable floating cells at $6,12,18$, and 24 h of duration (Fig. 3). The electron microscopy data show the ultra-structural characteristics of the Taheebo treated cultures revealing the presence of apoptotic cells characterized by the presence of peripheral condensation of the chromatin and membrane 'blebbing' (Fig. 2B), and of necrotic cells characterized by the presence of numerous lysosomes in the cytoplasm (Fig. 2B). Furthermore, the data presented in Fig. 3 reveal a timedependent increase in the incidence of a non-adherent (floating) cell population. 
Table I. Cell cycle regulation in ER+ human mammary carcinoma MCF-7 cells by Taheebo. ${ }^{\text {a }}$

\begin{tabular}{|c|c|c|c|c|c|c|}
\hline Treatment & $\begin{array}{c}\text { Dose } \\
(\mathrm{mg} / \mathrm{ml})\end{array}$ & $\begin{array}{c}\text { Duration } \\
\text { (h) }\end{array}$ & $\begin{array}{l}\text { G1 } \\
(\%)\end{array}$ & $\begin{array}{c}\mathrm{S}+\mathrm{G} 2 / \mathrm{M} \\
(\%)\end{array}$ & Ratio & $\begin{array}{l}\text { Modulation } \\
\text { (\% control) }\end{array}$ \\
\hline None & & 24 & 53.2 & 46.5 & 1.1 & - \\
\hline Taheebo & 0.75 & 24 & 59.9 & 39.9 & 1.5 & +36.4 \\
\hline None & & 48 & 63.1 & 36.7 & 1.7 & - \\
\hline Taheebo & 0.75 & 48 & 64.9 & 35.0 & 1.8 & +5.9 \\
\hline None & & 72 & 67.5 & 32.3 & 2.1 & - \\
\hline Taheebo & 0.75 & 72 & 60.9 & 39.1 & 1.5 & -28.6 \\
\hline
\end{tabular}

${ }^{\text {a}}$ Data expressed as arithmetic means from independent determinations in triplicate.

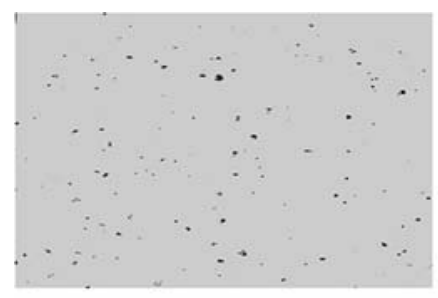

A

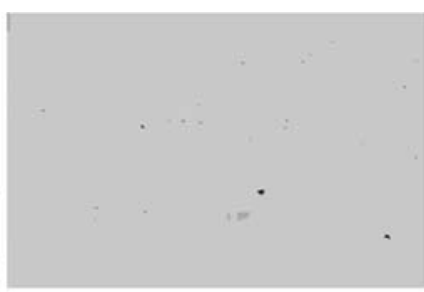

B
Figure 4. Inhibition of clonogenic growth of $\mathrm{ER}^{+} \mathrm{MCF}-7$ cells in response to $72 \mathrm{~h}$ of pre-treatment with $0.75 \mathrm{mg} / \mathrm{ml}$ of Taheebo. (A) Colonies in the untreated control. (B) Colonies in the Taheebo treated cultures at day 8 post-seeding.

Long-term growth inhibition of ER+ MCF-7 cells by Taheebo. The persistence of long-term growth inhibition in response to treatment with Taheebo was examined in the experiment presented in Fig. 4A and B. The colony counts obtained at the end of 11 days ( 3 day pre-treatment and 8 day clonogenic assay) revealed that relative to control cultures, mean colony number $419.0 \pm 6.4$ (Fig. 4A), the Taheebo treated cultures exhibited $88 \%$ inhibition mean colony number $50.3 \pm 2.9$, $\mathrm{p}<0.001$ (Fig. 4B), in the number of colonies formed.

Modulation of global gene expression profile of $\mathrm{ER}^{+} \mathrm{MCF}-7$ cells by Taheebo. The data presented in Table II examine the modulatory effect of Taheebo on global gene expression profiles in ER ${ }^{+}$MCF-7 cells. In Taheebo treated cells from among multiple genes functioning in the regulation of cell cycle progression, down-regulation of $\mathrm{S}$ phase specific PCNA, GSTE1 and OKL38, and G2 specific cyclin B and cdc2 resulted in a $>1.5$-fold reduction relative to that in untreated controls. In contrast, among multiple genes functionally involved in cellular apoptosis, the GADD45 gene family members and inactivators of the anti-apoptotic $\mathrm{BCl}-2$, such as BIM, HRK/BH3 and BBC3 were notably up-regulated to $>1.5$-fold, relative to those in untreated controls. Furthermore, tumor necrosis factor receptor super family (TNFRSF) genes responsible for apoptosis, and Calpain family genes responsible for necrosis were also up-regulated. Genes responsible for estrogen responsiveness such as ESR1 for ER- $\alpha$ were down-regulated, while genes such as CYP 1A1 and CYP 1B1, responsible for xeno-biotic metabolism, including that of estradiol were significantly up-regulated.

\section{Comparison of the data on gene expression by} microarray and real time PCR

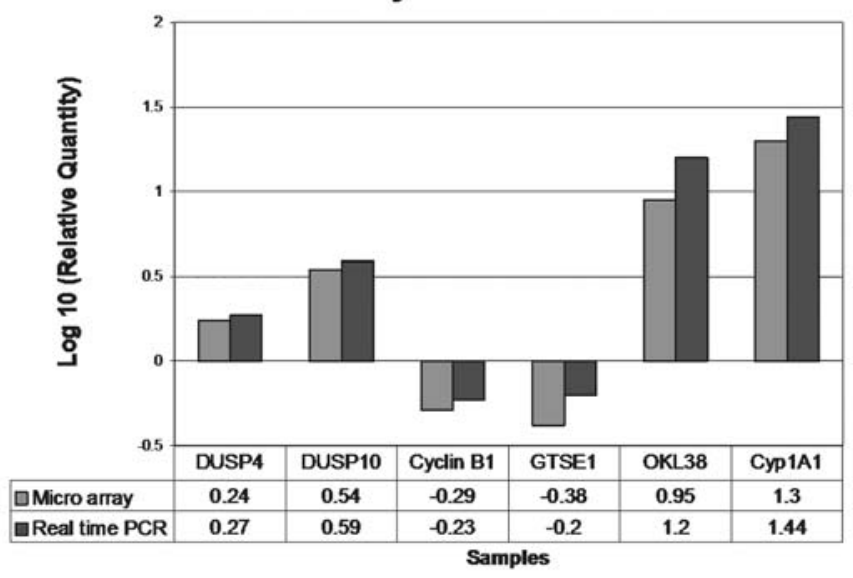

Figure 5. A comparison in modulation of gene expression using the microarray and real-time PCR assays.

The extent of modulation in the expression of selected genes was also confirmed using real-time PCR assay (Fig. 5).

\section{Discussion}

It is generally recognized that hormone responsive $\mathrm{ER}^{+}$ clinical breast cancers represent $\sim 80 \%$ of all sporadic cancer and exhibit a positive response to endocrine based therapeutic interventions $(1,2)$. However, long-term endocrine therapy with selective estrogen receptor modulators is frequently associated with reduced patient compliance, in part due to acquired drug resistance, or adverse systemic toxicity. These limitations emphasize a need to identify efficacious novel agents with minimal long-term toxicity.

The present study utilized the well-established human breast carcinoma derived $\mathrm{ER}^{+} \mathrm{MCF}-7$ cell culture model to examine the therapeutic efficacy of a natural plant extract, Taheebo, and to identify potential mechanistic leads for its efficacy on $\mathrm{ER}^{+}$clinical breast cancer.

The phenomenological evidence for the growth inhibitory effect of Taheebo on ER ${ }^{+}$MCF-7 cells is supported by the data that demonstrate a dose- and time-dependent inhibition of growth in response to treatment with Taheebo. These growth curves also identified optimum maximally cytostatic treatment for Taheebo in the present model system. 
Table II. Modulation of gene expression profiles in ER+ human mammary carcinoma MCF-7 cells by Taheebo. ${ }^{\mathrm{a}}$

\begin{tabular}{|c|c|c|c|c|c|}
\hline \multirow[t]{2}{*}{ Gene } & \multirow[t]{2}{*}{ Gene bank no. } & \multicolumn{2}{|c|}{ Fold Change } & \multicolumn{2}{|c|}{ Log Ratio } \\
\hline & & up & down & Log Ratio & Function \\
\hline
\end{tabular}

Cell cycle regulatory genes

GTSE1

NM_ 016426

PCNA

NM_002592

Cyclin B1

NM_031966

CyclinE1 NM_001238

CyclinE2 NM_057749

cdc2 NM_001786

Cyclin A2

NM_001237

RFC4

NM_002916

cdc20

NM_001255

Chk1

NM_005197

CCRK

NM_178432

cdk6

NM_001259

cdk2

p18

NM_001262

EXO1

NM_002692

NM_003686

OKL38

NM_013370

11.03

MCM6

NM_005915

$\operatorname{cdc} 25 \mathrm{~A}$

NM_001789

NM_198433

AURKA

NM_001255

$\operatorname{cdc} 20$

NM_032637

SKP2

POLA2

NM_002689

HCAP-G

NM_022346

$\operatorname{cdc} 7$

NM_003503

AMID

NM_032797

2.62

Caspase4

NM_033306

1.62

caspase 7

NM_033339

1.652

caspase8

NM_033356

1.643

AATK

PAWR

AK131529

GADD45G

NM_002583

1.8

1.47

3.47

$2.38 \quad-0.376$

1.96

$-0.29$

$1.96-0.29$

$1.6-0.2$

$3.22-0.5$

$2.56-0.4$

$2.77 \quad-0.44$

$1.56-0.19$

$1.85-0.27$

1.61

0.2

0.26

$2.0 \quad-0.296$

$1.50 \quad-0.18$

$1.88-0.275$

$1.81-0.26$

$1.51-0.182$

1.04

$1.639-0.21$

$1.75 \quad-0.24$

$1.85-0.27$

$1.85-0.27$

$1.96-0.29$

$1.89-0.276$

$1.69-0.226$

$1.85-0.267$

0.21

0.22

0.21

0.25

0.17

0.54

0.28

0.73
Homo sapiens $\mathrm{G} 2$ and $\mathrm{S}$ phase expressed 1 (GTSE1). DNA damage response. Signal transduction by p53 class mediator resulting in cell cycle arrest. Homo Sapiens proliferating cell nuclear antigen (PCNA), regulation of cell cycle; DNA replication

Regulation of cell cycle at G2/M Regulation of cell cycle at G1/S transition. Regulation of cell cycle at G1/S transition.

Cell division cycle 2 .

Mitotic G2 arrest. Regulators of CDK kinases. This cyclin binds and activates $\mathrm{CDC} 2$ and $\mathrm{cdK} 2$ at G1/S and G2/M transitions.

Replication factor $\mathrm{C} 4$

Cell division cycle homolog 20.

Checkpoint suppressor 1.

Cell cycle related kinase (CCRK); p42.

Cyclin dependent kinase 6.

Cyclin dependent kinase 2.

Cdk-4 inhibitor, p18.

$\varepsilon$ DNA polymerase activity DNA replication etc.

DNA binding exonuclease (5' to $3^{\prime}$ ) activity, interacts with

Msh2 which is involved in Mismatch repair.

Homo sapiens pregnancy-induced growth inhibitor, negative regulation of cell growth (akaBDG1)

Minichromosome maintenance deficient 6 DNA replication related function.

Regulate cyclin dependent protein kinase act. cdc25A DUSP control entry into and progression through $\mathrm{S}$ phase by dephosphorylating sites on cyclinE-cdk2 (Thr14,Tyr15).

Cell cycle regulated kinase that appears to be involved in microtubule formation/chromosome seggregation and stabilizes spindle pole etc. It interacts with multiple checkpoints in cell cycle. It is involved for nuclear movement prior to anaphase and chromosome segregation.

$\mathrm{S}$ phase kinase associated protein 2; It is an element for cycA-cdk2 $\mathrm{S}$ phase kinase

DNA directed polymerase- $\alpha$; DNA replication. Homo sapiens chromosome condensation protein $\mathrm{G}$. Mitotic cell cycle, chromosome condensation.

Negative regulation of cell proliferation. G1/S transition. cDc7 activity is essential for initiation of DNA replication.

Homo Sapiens apoptosis inducing factor (AIF) like mitochondrion - associated inducer of death (AMID).

Transcript variant $\mathrm{C}$, caspase 8 . Apoptosis associated tyrosine kinase.

PRKC, apoptosis WT1 regulator.

GADD 45 family A,B,G are responsible for growth suppression, induction of apoptosis through inhibition of NF- $\mathrm{B}$, up-regulation of c jun $\mathrm{N}$ terminal kinase (JNK). 
Table II. Continued.

\begin{tabular}{|c|c|c|c|c|c|}
\hline \multirow[t]{2}{*}{ Gene } & \multirow[t]{2}{*}{ Gene bank no. } & \multicolumn{2}{|c|}{ Fold Change } & \multicolumn{2}{|r|}{ Log Ratio } \\
\hline & & up & down & Log Ratio & Function \\
\hline TP53INP1 & NM_033285 & & 1.47 & -0.17 & $\begin{array}{l}\text { Its transcription is activated by p53. } \\
\text { It is responsible for G1 arrest and } \\
\text { p53 mediated apoptosis. }\end{array}$ \\
\hline CCAR1 & NM_018237 & & 1.55 & -0.19 & \\
\hline \multicolumn{6}{|l|}{ BCl-2 Family } \\
\hline BCL-2 & NM_000633 & & 1.53 & -0.19 & \\
\hline HRK/BH3 & NM_003800 & 3.75 & & 0.57 & $\begin{array}{l}\text { Activator of apoptosis. HRK regulates apoptosis } \\
\text { through inactivating BCL2 and BCLXL but } \\
\text { not with the death promoting Bcl } 2 \text { related } \\
\text { protein BAX, BAK or BCLXs. HRK interacts } \\
\text { with (BH3) domain with BCL2/BCL-XL. }\end{array}$ \\
\hline Bcl2like11 (BIM) & NM_138622 & 2.156 & & 0.33 & $\begin{array}{l}\text { Homo Sapiens apoptotic like facilitator. } \\
\text { BCL-2 like } 11 .\end{array}$ \\
\hline $\mathrm{BBC} 3$ & NM_014417 & 3.08 & & 0.49 & $\begin{array}{r}\text { Homo Sapiens BCL-2 binding component } 3 \\
\text { also known as PUMA. }\end{array}$ \\
\hline Athanogene1 & NM_004323 & 1.758 & & 0.245 & $\begin{array}{l}\text { BCL2 associated athanogene1. It binds and } \\
\text { enhances anti-apoptotic effects of BCL2 and } \\
\text { represents a link between growth factor } \\
\text { receptors and apoptotic mechanism. }\end{array}$ \\
\hline
\end{tabular}

Athanogene3 NM_004281 1.6

Tumor necrosis factor receptor super family genes

$\begin{array}{llll}\text { TNRSF1A } & \text { NM_001065 } & 2.44 & 0.387 \\ \text { TNFR SF6 } & \text { NM_000043 } & 1.67 & 0.22 \\ \text { TNFRSF21 } & \text { NM_014452 } & 1.677 & 0.22 \\ \text { Calpain family } & & & \\ \text { CAPN12 } & \text { NM_144691 } & 1.6 & 0.2 \\ \text { CAPN10 } & \text { NM_023083 } & 1.5 & 0.176 \\ \text { CAPN2 (m/II) } & \text { NM_001748 } & 1.46 & 0.17\end{array}$

Dual specific phosphatase (DUSP) family genes

\begin{tabular}{|c|c|c|c|c|c|}
\hline MAP2K6 & NM_002758 & & -4.786 & -0.68 & Extracellular signal regulated (ERK) kinase. \\
\hline MAP3K1/MEKK1 & ENST0000026477 & & -1.5 & -0.18 & Activates other MAP kinase signalling pathway. \\
\hline MAP3K2/MEKK2 & NM_006609 & & -1.47 & -0.18 & Phosphorylates and activates I $\kappa ß$ kinase. \\
\hline MAP3K10 & NM_002446 & 1.64 & & 0.21 & Induction of apoptosis via JUN pathway. \\
\hline MAP3K14 & NM_003954 & 1.8 & & 0.25 & $\mathrm{NF}-\kappa \mathrm{B}$ inducing kinase. \\
\hline DUSP2 & NM_004418 & & 2.63 & -0.42 & Dual specific phosphatase 2 . \\
\hline DUSP8 & NM_004420 & 2.2 & & 0.34 & Dual specific phosphatase 8 . \\
\hline DUSP4 & NM_001394 & 1.75 & & 0.24 & Dual specific phosphatase 4 . \\
\hline DUSP10 & NM_007207 & 3.53 & & 0.547 & Dual specific phosphatase 10. \\
\hline DUSP5 & NM_004419 & 2.02 & & 0.3 & Dual specific phosphatase 5 . \\
\hline DUSP 13 & NM_001007271 & 2.0 & & 0.3 & Dual specific phosphatase 13 . \\
\hline DUSP1 & NM_004417 & 1.8 & & 0.25 & Dual specific phosphatase 1 . \\
\hline DUSP 16 & NM_0030640 & 2.62 & & 0.41 & Dual specific phosphatase 16 . \\
\hline \multicolumn{6}{|c|}{ Estrogen responsive genes } \\
\hline ESR1 & NM_000125 & & 1.6 & -0.2 & Estrogen receptor- $\alpha$. \\
\hline CYP1A1 & NM_000499 & 19.77 & & 1.3 & \\
\hline CYP1B1 & NM_000104 & 7.95 & & 0.9 & \\
\hline
\end{tabular}

${ }^{a}$ Modulation in gene expression. Fold change represents comparison between Taheebo treated and untreated control group. Values for fold change represent means from independent triplicate sets of experiments. Genes in bold have been verified by real-time PCR.

TNFRSF family leads to apoptosis through MAPK/JNK and/or through Fas -associateed death domain (FADD).

Calpains, together with the lysosomal aspartyl proteases mediate necrotic cell death.

0.17 
The experiments designed to identify possible phenomenological leads for growth inhibition revealed that a short-term treatment of up to $72 \mathrm{~h}$ is associated with a progressive timedependent decrease in $\mathrm{G} 1: \mathrm{S}+\mathrm{G} 2 / \mathrm{M}$ ratio due to a modest $\mathrm{S}$ phase arrest. This is consistent with a progressive timedependent increase in the incidence of non-adherent (floating) cells. These data suggest that regulation of cell cycle progression and induction of cellular apoptosis/necrosis represent susceptible mechanistic pathways that are responsible for the observed effects of Taheebo.

The experiment was designed to examine the long-term effects of Taheebo using the clonogenic assay revealed that withdrawal of Taheebo retained its inhibitory effects for at least 8 days. These data identify possible leads that dose fractionation achieved by discontinuous treatment reduces long-term toxicity without compromising the efficacy.

The experiment utilizing micro-array based global gene expression profiling identified important leads for possible molecular targets and mechanistic pathways critical for the efficacy of Taheebo. Overall, the micro-array assay examined the gene expression profiles of 43,376 genes, using the $44 \mathrm{~K}$ gene chip. About 1,600 genes (3.7\%) exhibited down-regulated expression, 1,900 genes (4.4\%) exhibited up-regulated expression, and 40,000 genes $(92.2 \%)$ remained essentially unchanged. It is clear from the data obtained from selected genes relevant to growth, proliferation, apoptosis and metabolism of ER ${ }^{+} \mathrm{MCF}-7$ cells in the culture that Taheebo modulates the expression of multiple genes that are involved in these biological processes. The modulation in gene expression profiles essentially correlated with the cell cycle regulation and induction of initial stages of cellular apoptosis. Thus, with regard to $\mathrm{S}$ phase arrest, it is noteworthy that genes such as PCNA and GTSE1 were down-regulated. These genes play a role in DNA synthesis and replication at the $S$ phase of the cell cycle (16). In addition, the GADD 45 family of genes was also substantially up-regulated in response to the treatment with Taheebo. This gene family is involved in the regulation of growth, DNA repair, and induction of apoptosis (17). Interestingly, OKL38 gene, also known as the human bone marrow stromal cell derived growth inhibitor (BDGI), exhibited a robust 12-fold up-regulation. This gene is known to induce $S$ phase arrest with subsequent initiation of apoptosis $(18,19)$. The genes responsible to inactivate antiapoptotic BCL-2, such as BIM, HRK/BH3 and BBC3 exhibited 2.16-, 3.75-, and 3.08-fold up-regulation, respectively. These genes are also known to arrest the cells in the $S$ phase and initiate apoptosis $(19,20)$. These data on the modulated expression of OKL38, BIM, HRK/BH3 and BBC3 genes taken together, suggest that initiation of the apoptotic process by Taheebo is in part due to reduced function of the anti-apoptotic $\mathrm{BCl}-2$ gene. In this context it is also noteworthy that consistent with the presence of necrotic cells in Taheebo treated cultures, the Calpain family genes (21) were up-regulated to $>1.5$-fold, relative to those in untreated controls.

Regarding the effect of Taheebo on ER signaling, it was observed that Taheebo treatment up-regulated the dual specific phosphatase (DUSP) gene family is responsible for negative regulation of MAPK signaling (22-24). These data indicate that Taheebo suppresses the proliferation of $\mathrm{ER}^{+} \mathrm{MCF}-7$ cells via its effect on the ligand independent AF-1 domain that is critical for the ER signaling pathway. Consistent with the possible efficacy of Taheebo via ER signaling, observed downregulation of cyclin A and cdk2 is suggestive of an inhibition of phosphorylation of ER N-terminal AF-1 domain $(25,26)$. This interpretation provides proof of a principle mechanistic lead for the possible anti-estrogenic effects of Taheebo components at high cytostatic doses via the ER signaling pathway.

With regard to estrogen responsive genes, it is noteworthy that Taheebo treatment resulted in 1.6-fold down-regulation of ESR1, the gene for ER- $\alpha$, while ESR2, the gene for ER- $\beta$, remained essentially unaltered. Although, CYP 1A1 and CYP 1B1 genes are primarily involved in xeno-biotic metabolism, they are also responsible for cellular metabolism of estradiol leading to the generation of $\mathrm{C} 2-, \mathrm{C} 4-$, and $\mathrm{C} 16 \alpha-$ hydroxylated metabolites capable of modulating the growth of MCF-7 cells (27-29). Similar to the MCF-7 cells, modulation of cellular metabolism of estradiol was noted in the 184B5/HER cell culture model for $\mathrm{ER}-/ \mathrm{HER}-2^{+}$pre-invasive comedo ductal carcinoma in situ (30).

All the targets and pathways discussed above are well recognized in the growth, cellular proliferation and tumorigenecity of ER ${ }^{+}$MCF-7 cells $(12,13)$. Furthermore, experimental modulation in these pathways in response to treatment with selective estrogen receptor modulators is associated with growth inhibition of MCF-7 cells both in in vitro cell cultures as well as in in vivo xeno-transplant models for tumorigenecity via multiple mechanisms $(12,13,22,23,25,26)$. These data taken together, suggest that the mode of action of Taheebo is similar to that of a selective estrogen receptor modulator. In this context it is noteworthy that published studies with Tamoxifen on $\mathrm{ER}^{+} \mathrm{MCF}-7$ cells demonstrated that in addition to the ER-dependent mechanism, the selective estrogen receptor modulator Tamoxifen alters cellular metabolism of estradiol to favor the generation of anti-proliferative metabolites (31-33), and influence cell cycle progression in MCF-7 cells via cdk-4/6 and p53-dependent pathways $(12,13,34)$.

With regard to the identification of molecular target(s) and mechanistic pathway(s) for the efficacy of Taheebo, it needs to be recognized that non-fractionated aqueous extract of Taheebo used in the present study contains a mixture of several mechanistically distinct constituents including furanonaphthoquinones, quinines, naphthoquinones and flavonoids (35-37). Naphthofurandione, a major component of Taheebo, has selective anti-tumor effects on a variety of human carcinoma derived cell lines (35-38). It is therefore, conceivable that the efficacy for growth inhibition by Taheebo, as evidenced by the present data, is due to interactive effects of multiple water soluble components from Taheebo.

In conclusion, the outcome of the present study validates a human tissue derived cell culture approach to evaluate the efficacy of non-fractionated herbal medicinal extracts and to identify clinically translatable mechanistic leads for alternative therapy of $\mathrm{ER}^{+}$clinical breast cancer.

\section{Acknowledgements}

Major funding for this research was provided by philanthropic contributions to American Foundation for Chinese 
Medicine from the family of Daniel and Kathleen Mezzalingua, the family of Haken and Marie Ledin and Issac and Laura Perlmutter Fund.

\section{References}

1. Lippman ME: Efforts to combine endocrine and chemotherapy in the management of breast cancer: do two and two equal three? Breast Cancer Res Treat 3: 117-127, 1983.

2. Early Breast Cancer Trialist's Collaborative Group (EBCTG): Effects of chemotherapy and hormonal therapy for early breast cancer on recurrence and 15 years survival: An overview of the randomized trials. Lancet 365: 1687-1717, 2005.

3. Rock E and DeMichele A: Nutritional approaches to late toxicities of adjuvant chemotherapy in breast cancer survivors. J Nutr 133: 3785s-3793s, 2003.

4. Oudin C, Bonnetain F, Boidot R, et al: Patterns of loss of heterozygosity in breast carcinoma during neo-adjuvant chemotherapy. Int J Oncol 30: 1145-1155, 2007.

5. Molassiotis A, Scott JA, Kearney N, et al: Complementary and alternative medicine use in breast cancer patients in Europe. Support Care Cancer 14: 260-267, 2006.

6. Tindle HA, Davis RB, Phillips RS, et al: Trends in the use of complementary and alternative medicine by US adults: 19972002. Altern Ther Health Med 11: 42-49, 2005.

7. Helyer LK, Chin S, Chui BK, et al: The use of complementary and alternative medicines among patients with locally advanced breast cancer - a descriptive study. BMC Cancer 6: 39-46, 2006.

8. Matthews AK, Sellergren SA, Huo D, et al: Complementary and alternative medicine use among breast cancer survivors. J Altern Complement Med 13: 555-562, 2007.

9. Park BS, Lee HK, Lee SE, et al: Antibacterial activity of Tabebuia impetiginosa Martius ex DC (Taheebo) against Helicobacter Pylori. J Ethnopharmacol 105: 255-262, 2006.

10. Park BS, Kim JR, Lee SE, et al: Selective growth-inhibiting effects of compounds identified in Tabebuia impetiginosa inner bark on human intestinal bacteria. J Agri Food Chem 53: 1152-1157, 2005.

11. Son DJ, Lim Y, Park YH, et al: Inhibitory effects of Tabebuia impetiginosa inner bark extract on platelet aggregation and vascular smooth muscle cell proliferation through suppressions of arachidonic acid liberation and ERK1/2 MAPK activation. J Ethnopharmacol 108: 148-151, 2006.

12. Lippman ME, Osborne CK, Knazek R and Young N: In vitro model systems for the study of hormone dependent breast cancer. N Engl J Med 296: 154-159, 1977.

13. Jordan VC: Historical perspective on hormonal therapy of advanced breast cancer. Clin Ther 24: A3-A16, 2002.

14. Hsieh TC, Lu X, Guo J, et al: Effects of herbal preparation Equigard on hormone responsive and hormone refractory prostate carcinoma cells. Int J Oncol 20: 681-689, 2002.

15. Livak KJ and Schmittgen TD: Analysis of relative gene expression data using real-time quantitative PCR and the 2(-Delta Delta C (T)) method. Methods 25: 402-408, 2001.

16. Monte MR, Benetti R, Buscemi G, et al: The cell cycleregulated protein human GTSE-1 controls DNA damageinduced apoptosis by affecting p53 function. J Biol Chem 278: 30356-30364, 2003.

17. Yin F, Bruemmer D, Blaschke F, et al: Signaling pathways involved in induction of GADD45 gene expression and apoptosis by troglitazone in human MCF-7 breast carcinoma cells. Oncogene 23: 4614-4623, 2004.

18. Huynh $\mathrm{H}, \mathrm{Ng} \mathrm{CY}$, Ong CK, et al: Cloning and characterization of a novel pregnancy-induced growth inhibitor in mammary gland. Endocrinology 142: 3607-3615, 2001.

19. Wang T, Xia D, Li N, et al: Bone marrow stromal cell-derived growth inhibitor inhibits growth and migration of breast cancer cells via induction of cell cycle arrest and apoptosis. J Biol Chem 280: 4374-4382, 2005.
20. Concannon CG, Koehler BF, Reimertz C, et al: Apoptosis induced by proteasome inhibition in cancer cells: predominant role of the p53/PUMA pathway Oncogene 26: 1681-1692, 2007.

21. Artal-Sanz M and Tavernarakis N: Proteolytic mechanisms in necrotic cell death and neuro-degeneration. FEBS Lett 579: 3287-3296, 2005.

22. Frigo DE, Basu A, Nierth-Simpson EN, et al: p38 Mitogenactivated protein kinase stimulates estrogen mediated transcription and cell proliferation through the phosphorylation and potentiation of the $\mathrm{p} 160$ co-activator glucocorticoid receptor interacting protein1. Mol Endocrinol 20: 971-983, 2006.

23. Creighton CJ, Hilger AM, Murthy S, et al: Activation of mitogen-activated protein kinase in estrogen receptor alphapositive breast cancer cells in vitro induces an in vivo molecular phenotype of estrogen receptor alpha-negative human breast tumors. Cancer Res 66: 3903-3911, 2006.

24. Jeffrey KL, Camps M, Rommel C and Mackay CR: Targeting dual-specificity phosphatases: manipulating MAP kinase signaling and immune responses. Nature Rev Drug Disc 6: 391-403, 2007.

25. Rogatsky I, Trowbridge JM and Garabedian MJ: Potentiation of human estrogen receptor alpha transcriptional activation through phosphorylation of serines 104 and 106 by the cyclinA-cdk2 complex. J Biol Chem 274: 22296-22302, 1999.

26. Trowbridge JM, Rogasky IO and Garabedian MJ: Regulation of estrogen receptor transcriptional enhancement by the cyclin A/Cdk2 complex. Proc Natl Acad Sci 94: 10132-10137, 1997.

27. Yager JD and Davidson NE: Estrogen carcinogenesis in breast cancer. N Engl J Med 354: 270-282, 2006.

28. Schneider J, Huh MM, Bradlow HL and Fishman J: Anti-estrogen action of 2-hydroxyestrone on MCF-7 human breast carcinoma cells. J Biol Chem 259: 4840-4845, 1984.

29. Lottering ML, Haag M and Seegers JC: Effects of 17ß-estradiol metabolites on cell cycle events in MCF-7 cells. Cancer Res 52: 5926-5932, 1992.

30. Telang NT, Katdare M, Bradlow HL and Osborne MP: Cell cycle regulation, apoptosis and estradiol metabolism: Novel end point biomarkers for human breast cancer prevention. J Clin Ligand Assays 23: 130-137, 2000.

31. Suto A, Bradlow HL, Kubota T, et al: Alteration in proliferative and endocrine responsiveness of human mammary carcinoma cells by prototypic tumor suppressing agents. Steroids 58: 215-219, 1993.

32. Telang NT, Bradlow HL and Osborne MP: Effect of Tamoxifen on mammary preneoplasia: relevance to chemopreventive intervention. Cancer Detect Prev 18: 313-321, 1994.

33. Osborne MP: Chemoprevention of breast cancer. Surg Clin North Am 79: 1207-1221, 1999.

34. Cover CM, Hsieh SJ, Cram EJ, et al: Indole-3-carbinol and tamoxifen co-operate to arrest cell cycle of MCF-7 human breast cancer cells. Cancer Res 59: 1244-1251, 1999.

35. Queiroz ML, Valadares MC, Torello CO, et al: Comparative studies of the effects of Tabebuia avellanedae bark extract and $\beta$-lapachone on the hematopoietic response of tumor-bearing mice. J Ethnopharmacol 117: 228-235, 2008.

36. Ueda S, Umemura T, Dohguchi K, et al: Production of anti-tumorpromoting furano-naphthoquinones from Tabebuia avellanedae in cell cultures. Phytochemistry 36: 323-325, 1994.

37. Park BS, Lee KG, Shibamoto T, et al: Antioxidant activity and characterization of volatile constituents of Taheebo (Tabebuia impetiginosa Martius ex DC). J Agri Food Chem 51: 295-300, 2003.

38. Ueda S, Tokuda H, Hirai K, et al: A novel antitumor compound 2-(1-hydroxyethyl)-5-hydroxynaptho [2, 3-b] furan -4, 9-dione and anti-tumor agents comprising this compound. United States of America, Patent \# 5,663,197, 1997. 